\title{
PERFORMANCE ANALYSIS OF AM-FM ESTIMATORS
}

\author{
Jitendra Kumar Gupta. S. Chandra Sekhar and T.V. Sreenivas* \\ Dept. of Electrical Communication Engg., \\ Indian Institute of Science, \\ Bangalore - 560 012, INDIA. \\ *e-mail:tvsree@ece.iisc.ernet.in
}

\begin{abstract}
We address the problem of decomposing a handpass signal into amplitude arid frequency modulated components (AM and FM respectivelyj. Several estimators have been proposed in literature. each of which works under specific assumptions. In this paper, we perform a comparative study of some popular techniques hy studying their performance with changes in the modulation parameters and the tradeoffs involved. We also study their performance in the presence of handpass white Gaussian noise.
\end{abstract}

\section{INTRODUCTION}

Most practical signals, such as speech, audio. biomedial signals are nonstationary in nature and have time-varying spectral characteristics. The time-varying spectral characteristic is usually, a direct consequence of the signal generation process. The spectral variability with time can be decomposed into amplitude variability and frequency variability. Most sources convey information through modulations of amplitude (amplitude modulation or AM) and frequency (frequency modulation or FM) of a steady camer which serves as a means of transporting the information contained in the modulations. The basic and simplest signal processing model of such a. source is therefore an AM-FM combination.

Given a bandpass signal, it is possible to decompose it into an AM-FM combination. Un the other hand, given an AM-FM combination, we can synlhesize a handpass signal. Several such AM-FM Combinations can be used to represent complex signals such as speech and audio. Decomposing a bandpass signal into its AM and FM parts has been addressed by many researchers and a number of techniques have been published in the literature. Each technique works under specific assumptions. Also, the performance of these techniques in the presence of handpass noise has not been addressed.

The present work is motivated by the following questions:
I. How do the techniques perform for large camer frequencies and large frequency deviations'?

2. How does noise affect the performance of these methods?

\section{SIGNAL MODEL}

The signal model is given as:

$$
x(t)=A(t) \cos \left(2 \pi f_{c} t+2 \pi k_{f} \int_{-\infty}^{t} m(\tau) d \tau\right)
$$

where $A(t)$ is the amplitude modulation. The phase modulation component, represented by $\phi(t)$ is given by $\phi(t)=$ $2 \pi f_{c} t+2 \pi k_{f} \int_{-\infty}^{t} m(\tau) d \tau$. The 'instantaneous frequency'(IF), $f_{i}(t)$ is defined as $f_{i}(t)=\frac{1}{2 \pi} \frac{d \phi(t)}{d t}=f_{c}+k_{f} m(t)$. which shows modulation of frequency by the signal $m(t)$. In terms of the angular frequency, $\omega_{i}(t)=2 \pi f_{i}(t)$. The methois that will bc discussed use the discrete-time version of the above signal(for implementation purposes), which, with normalized sampling period of unity is given as

$$
x[n]=A[n] \cos \left(2 \pi f_{c} n+2 \pi k_{f} \sum_{l=-\infty}^{n} m[l]\right)
$$

$A[n]$ is the discrete-time AM and the IF is given as $f_{i}[n]=$ $\frac{1}{2 \pi}(\phi(n)-\phi(n-1))=f_{c}+k_{f} m[n]$. The angular frequency is given by $\omega_{i}[n]=2 \pi f_{i}[n]$.

\section{METHODS FOR AM-FM DECOMPOSITION}

Of the several methods that exist, we have chosen the following popular ones for a comparative study:

1. Auditory Motivated AM-FM Decomposition [1],

2. Teager Energy based algorithms [2],

3. Positive time-Frequency distrihution approach $[3,6]$.

4. Hilbert transform approach 141. 


\section{Speech Processing / 955}

In llic subsections that follow, we briefly review these methods.

\subsection{Auditory Motivated AM-IM decomposition}

I h is method purely relies on onty the amplitude envelope, i.c., Ilic magnitude of the ottput of a linear lilter (characterized hy impulse response, $f_{l}[n]$ ), by exploiting the propcity of filter transchuction, i.e., the linear filler output can hc obtained approximately by sweeping $\omega[n]$ through the filter's transfer function [I]. The amplitude envelope of the instantaneous output is given by the approximation $y[n] \approx$ $0.5 A[n] . H(w[n])$. Only the magnitude of the filter response $H(\omega)$ is used $(H(\omega)$ is a zero-phase filter). To solve for the $\mathbf{A M}$ and $\mathbf{P M}$ we needatleast two fitter outputs. A pair of bandpass filters are used. Two kinds of filters are commonly uscd - Piccowise-linear filters and Gaussian filters.

For two Piecewise-linear filters, $H_{1}(\omega)=a_{1} \omega+b_{1}$ and $H_{2}(\omega)=a_{2} \omega+b_{2}$. If the input is $A[n] \cos (\omega[n])$, the outputs can he approximated as $y_{1}[n] \approx a_{1} A[n] \omega[n]+b_{1} A[n]$ and $y_{2}[n] \approx a_{2} A[n] \omega[n]+b_{2} A[n]$. These equations can be solved for $A[n]$ and $\omega[n]$

The assumptions are that the $\mathbf{A M}$ is slowly varying

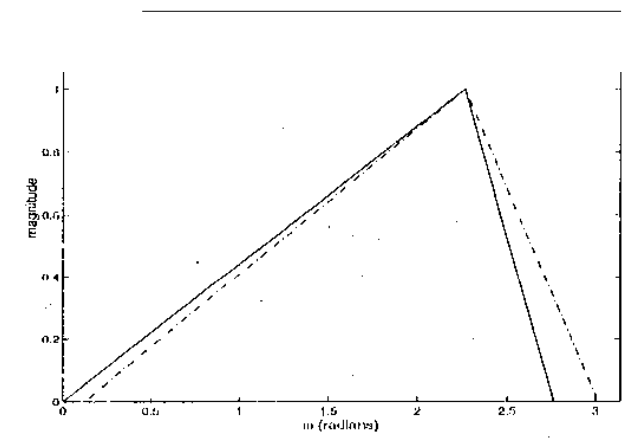

Fig. 1. Piecewise-linear auditory filters used for AM-PM demodulation

(bandwidth much less than the carrier frequency, $f_{c}$ ) and that the FM deviation is limited lo the region where both the bandpass filters have posilive or negative slope (a strict requirement for piecewise linear filters). If there is apriori knowledge of the center frequency, frequency deviation and bandwidth of AM, then appropriate fillers can he designed to perform AM-PM decomposition Otherwisc, we need to use a hank of overlapping bandpass fillers (which represent the auditory filterbank front-end) lo filter the input signal. The outputs of filters with the dominant energy (implying coincidence of the fi]ter spectra with input signal spectrum) can he used lo perform AM-FM decomposition.

In this work. we assumed apriori information about $f_{c}$ and frequency swing. Two filters used in the simulations are shown in Fig. I. We preler luese to those proposed in [1] because these can handle lager swings in frequency. For smatler swings in frcquency. both yield identicat results.

\subsection{Teager Energy Based Algorithms}

Of recent interest has been the use of 'leager energy for AMFM decomposition [2]. The energy opcrator (denoted by $\Psi\{x[n]\}$ on a signal $x[n]$ is definced as $\Psi\{x[n]\})=x^{2}[n]-$ $x[n-1][x\{n+1]$. There are two popular algorithms namely, DESA-I and DESA-2 (DESA stands for Discrete Finergy Separation Algorithm). The cxprcssions for $\mathbf{A M}$ and $\mathbf{F M}$ using llic DESA-I algorithm are given hy

$$
\begin{aligned}
& |a[n]| \approx \sqrt{\frac{\Psi\{x[n]\}}{1-\left(\xi_{1}[n]\right)^{2}}} \\
& \omega_{i}[n] \approx \cos ^{-1}(1-\xi[n])
\end{aligned}
$$

where $y[n]=x[n]-x[n-1]$ and $\xi_{1}[n]=\frac{\Psi\{y[n]\}+\Psi\{y[n+1]\}}{4 \Psi\{y[n]\}}$. DESA-1 algorithm can estimate IF such that $0<\omega_{i}[n]<\pi$.

The expressions for AM and FM using the DESA-2 algorithm are given by

$$
\begin{aligned}
|a[n]| & \approx \frac{2 \Psi\{x[n]\}}{\sqrt{\Psi}\{z[n]\}} \\
\omega_{i}[n] & \approx 0.5 \cos ^{-1}\left(1-\xi_{2}[n]\right)
\end{aligned}
$$

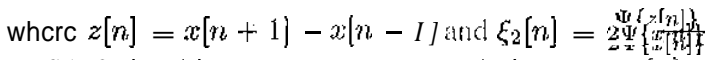
DESA-2 algorithm can estimate $\mid F$ such that $0<w_{i}[n]<$ $\frac{\pi}{2}$. Implementation details are discussed in $|2|$. Both these algorithms require that Ilic $\mathbf{A M}$ be very slowly varying and that frequency deviation of the signal across the carrier frequency be very small.

\subsection{Positive Time-frequency Distribution Approach}

In this approach, a positive time-Jiequency distribution(PTFD), $\Gamma(t, \omega)$ is used lo estimate llic I'M followed by $\mathbf{A M}$ estimallion using coherent time-varying demodulation [3]. CohenPosch positive time-frequency distribution $[6]$ is designed such that the time and frequency marginals, iustantaneous frequency property arc satisfied. Fnr an AM-FM combination signal, computing the average lirequency of the PTFD [5] at each time-instant gives the FMi.c.,

$$
\omega_{i}(t)=\frac{\int_{0}^{\infty} \omega P(t, \omega) d \omega}{\int_{0}^{\infty} P(t, \omega) d \omega}
$$

Using this, the instantaneous phase is given as $\phi(t)=\int_{\ldots \infty}^{l} \omega_{i}(\tau) d \tau$ is computed. The in-phase and quadrature components of 
TENCON 2003/956

the AM are obtained as:

$$
\begin{aligned}
& A_{i}(t)=\int_{-\infty}^{+\infty} x(\tau) \cos (\phi(t)) h_{l p}(t, \tau) d \tau \\
& A_{q}(t)=\int_{-\infty}^{+\infty} x(\tau) \sin (\phi(t)) h_{l p}(t, \tau) d \tau
\end{aligned}
$$

where $h_{l p}(t, \tau)$ is the time-varying impulse response of a low-pass filter with varying cutoff frequency $\omega_{i}(t)$ and passband gain equal to two. The AM is obtained by combining these results as $A(t)=h-T)+A_{i}(t)$. The FTFD has to be obtained iteratively staning with the spectrogram. Spectrogramcomputation depends upon the choice of a window. As a result, the window length plays a crucial role in limiting the performance of the algorithm. The method yields satisfactory results as long as the assumption that the signal is nearly stationary within the window holds.

\subsection{Hilbert Transform Approach}

This is perhaps, the oldest of techniques for AM-FM separation [4], Given a signal, $x(t)$, we compute its Hilbert transform $\widehat{x}(t)$. The analytic signal is defined as $c(t)=$ $x(t)+\jmath \widehat{x}(t)=a(t) e^{j \phi(t)}$, where $a(t)$ is the AM and $\phi(t)$ is the phase modulation component. The FM can he obtained as $f_{i}(t)=\frac{1}{2 \pi} \frac{d \phi(t)}{d t}$. The conceptual paradox associated with this method is that to obtain time-varying characteristics of the signal, which are local in nature, we use the Hilbert transform which is computed on a global basis.

For implementation purposes, we use the discrete-time Hilbert transform.

\section{PERFORMANCEANALYSIS}

The focus of the present study is to understand, by simulations, the limitations of these techniques for AM-FM decomposition for different carrier frequencies, frequency deviations and presence'of noise.

\subsection{Effect of Carrier Frequency and Frequency Devia- tion}

For the noise-free case, extensive simulations were carried out hut only results for the sinusoidal AM, and sinusoidal FM Combination are discussed due to lack of space.

1024 samples of an AM-FM combination were generated. 1.5 cycles of a sinusoid was used as the AM with $25 \%$ modulation depih $\left(\mu_{0}\right)$. The FM consisted of $\mathbf{3}$ cycles of a sinusoid with a frequency deviation (Af). In relation to the carrier frequency $f_{c}$, this is specified by the ratio $\mu_{f}=\boldsymbol{Y}$. The results of two experiments are reported here - performance as a function'of frequency deviation for a fixed carrier frequency (Experiment 1 ) and performance as
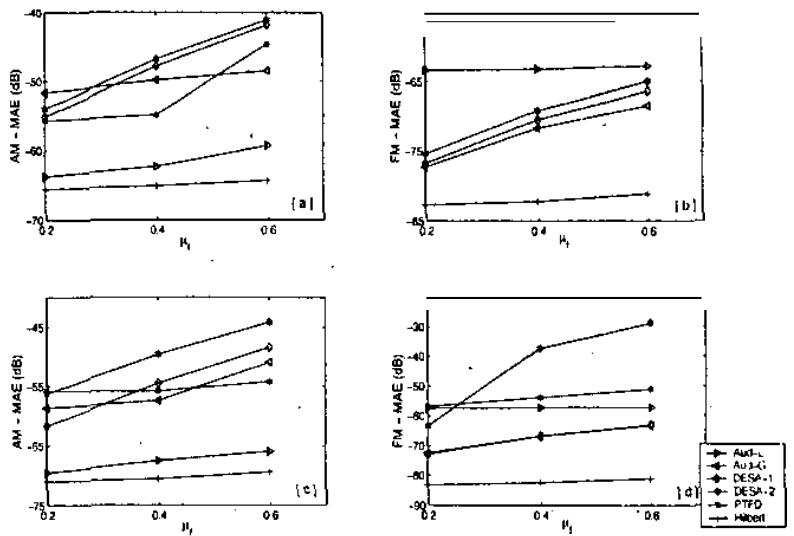

Fig. 2. Performance analysis as a function of $\mu_{f}$ for $f_{\mathfrak{c}}=$ $0.1 \mathrm{~Hz}$ (top row) and $f_{\mathrm{c}}=0.2 \mathrm{~Hz}$ (bottom row). [a];[c]AMMAE $(d B)$ and [b],[d]FM-MAE $(d B)$.

a function of carrier frequency for a fixed frequency deviation (Experiment 2). Expt. I was performed with $f_{\mathrm{c}}=0.1 \mathrm{~Hz}$, $\mu_{f}=0.2,0.4,0.6$. and $f_{c}=0.2 \mathrm{~Hz}, \mu_{f}=0.2,0.4,0.6$. The error measure used was the Mean Absolute Error (MAE) and defined as

$$
M A E_{\theta}=\frac{1}{N} \sum_{n}|\theta[n]-\hat{\theta}[n]|
$$

where $\theta$ is either the AM or FM and $\hat{\theta}$ denotes its estimate, giving respectively the MAE in AM and FM denoted by AM-MAE and FM-MAE. These are plotted in decibel $(\mathrm{dB})$ scale in Figs. 2 and 3.

From Fig. 2, we conclude that

1. All methods exhibit an increase in error with modulation depth for a fixed carrier frequency.

2. The maximum increase in error with modulation depth for a fixed $f_{c}$ is exhibited by DESA algorithms.

3. Consistently, DESA-I outperforms DESA-2.

4. Amongst auditory filtering based AM-FM separation. Piecewise-linear filters consistently outperform Gaussian filters in AM estimation. The reverse is true for FM estimation.

5. For the PTFD approach. with changes in carrier frequency and modulation depths, the FM estimation does not suffer and shows only marginal degradation. However, AM estimation degrades.

6. For low carrier frequencies and large modulation depths. the Hilben transform approach is the one that has 


\section{Speech Processing/ 957}
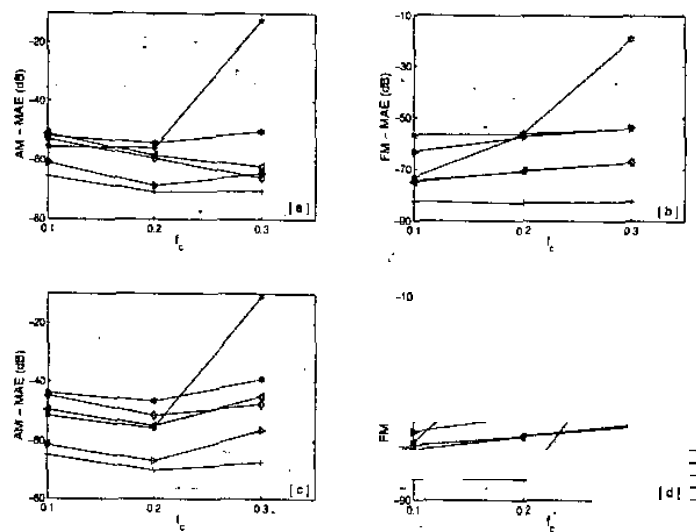

$-10$

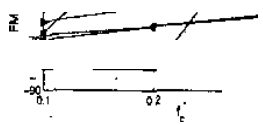

Fig. 3. Performance Analysis as a function of $f_{c}$ for $\mu_{f}=$ $25 \%$ (top row) and $\mu_{\%}=50 \%$ (bottom row). [a], $[c] A M$ $M A E(d B)$ and [b],[d]FM-MAE $(d B)$.

least error in both $\mathrm{AM}$ and FM. It is surprising to notc that the Hilbert transform approach that computes local information on a global basis has the least error unlike specifically designed tools to extract local information such as the other methods discussed here.

From Fig. 3, we conclude that

I. The Hilbert transform approach offers the best performance in AM estimation for small-to-large carriers with small-to-large modulation depths. The FM performance suffers for high carriers with high modulation depths.

2. Piecewise-Iinear.filters outpefform Gaussian filters in FM estimation' even for high carriers with large or small modulation depths. The reverse is true for AM estimation. The same conclusion was drawn from Fig. 2

3. The PTFD based AM estimation has high errors for smali/large modulation depths for large carriers. FM estimation however does not face similar problems.

4. DESA-I. consistently, outperforms DESA-2.

\subsection{Effect of Noise}

We study the effect of bandpass white Gaussian noise. Since AM-FM signals are essentially bandpass in nature, we consider the effects of only in-band noise. Sample realizations from a whitc Gaussian noise process were generated and bandpass filtered to give bandpass white noise. The bandpass region was selected as the frequency zone in which
$95 \%$ of the signal energy is concentrated. The bandpass filtered noise was suitably scaled to achieve a desired SNR. We consider two different AM-FM combinations

I. Sinusoidal AM, $A[n]=1+0.25 \cos \left(2 \pi \frac{1.5 n}{N}\right)$, sinusoidal FM, $f_{i}[n\}=0.18+0.045 \sin \left(2 \pi \frac{3 n}{N}\right), 0 \leq n \leq$ $N-1$.

2. Exponential AM, $A[n]=e^{\ln (0.1) \frac{\mathrm{n}}{N}}$, linear FM, $f_{i}[n]=$ $0.1+\frac{0.1 n}{N}, 0 \leq n \leq N-1$.

The parameters were chosen such that in the absence of noise, all techniques perform nearly identically. For each combination, SNR was varied from $4 \mathrm{~dB}$ to $32 \mathrm{~dB}$ in steps of 4dB. For each SNR, 40 Monte-Carlo realizations were used to obtain the average MAE measure. Finite data can cause large errors at the ends of the window. Hence, they were ignored in computing the error measure. Otherwise, the error measure will be severely biased. The results are tabulated in Tables. I and 2(values rounded to the nearest integer). One common trend exhibited by all of them is the decrease in error with increase in SNR. This is natural and expected. The following conclusions are in order:

I. At very low SNRs, the PTFD approach offers the best performance for all combinations considered and hence is more robust.

2. DESA-I performs better than DESA-2 even in the presence of noise

3. Almost all techniques show a linear decrease in error with increase in SNR. For every one-dB rise ih SNR, the error reduces by roughly one- $d B$ but for Hilbert transform where the error initially follows this rule but saturates for very high SNRs. We call this, the 'one-dB rule'.

4. At high SNRs, owing to their simplicity, the DESA algorithms may he preferred. At low SNRs. the PTFD approach is preferred though computationally more complex.

It must also he noted that if some apriori information is available about the smoothness of the AM and/or FM, then the estimates given by any of these methods can be further improved by filtering(linear or nonlinear depending on the nature of estimation errors). For example. for IF with dis. continuities (nol reported here). Hilbert transform approaches yield estimates with spiky errors which can be reduced using nonlinear filters.

\section{CONCLUSIONS}

In this paper, we studied the performance of different AMFM decomposition methods with and without noise. In the 


\section{TENCON 2003/958}

Tahle 1. Performance analysis for sinusoidal AM and sinusoidal FM, Mean Absolute Error(dB) in AM and FM as a function of SNR(dB). (T1:Piecewise-linear auditory filters, T2:Gaussian auditory filters, T3:DESA-1,T4:DESA-2,T5:PTFD, T6: Hilbert transform)

\begin{tabular}{|c||c|c|c|c|c|c||c|c|c|c|c|c|}
\hline \multicolumn{1}{|c||}{} & \multicolumn{9}{c|}{ AM-MAE(dB) } & \multicolumn{6}{c|}{ FM-MAE(dB) } \\
\hline SNR & T1 & T2 & T3 & T4 & T5 & T6 & T1 & T2 & T3 & T4 & T5 & T6 \\
\hline 4 & -09 & +00 & -09 & -09 & -10 & -09 & -30 & -34 & -33 & -33 & -40 & -35 \\
\hline 8 & -13 & +03 & -13 & -13 & -13 & -13 & -39 & -37 & -39 & -37 & -45 & -40 \\
\hline 12 & -17 & -06 & -17 & -17 & -17 & -17 & -44 & -41 & -43 & -44 & -50 & -44 \\
\hline 16 & -21 & -19 & -21 & -21 & -21 & -21 & -48 & -45 & -48 & -45 & -53 & -48 \\
\hline 20 & -25 & -24 & -25 & -25 & -25 & -25 & -52 & -49 & -52 & -49 & -55 & -52 \\
\hline 24 & -29 & -30 & -29 & -29 & -29 & -29 & -55 & -53 & -56 & -53 & -56 & -57 \\
\hline 28 & -33 & -34 & -33 & -33 & -33 & -33 & -57 & -57 & -59 & -57 & -56 & -60 \\
\hline 32 & -36 & -37 & -36 & -36 & -36 & -36 & -56 & -59 & -61 & -58 & -55 & -63 \\
\hline
\end{tabular}

Table 2. Performance analysis for exponential AM andlinear FM, Mean Absolute Error(dB) in AM and FM as a function of SNR(dB). (T1:Piecewise-linear auditory filters, T2:Gausșian auditory filters, T3:DESA-1,T4:DESA-2,T5:PTFD, T6:Hilbert transform)

\begin{tabular}{|c||c|c|c|c|c|c||c|c|c|c|c|c|}
\hline \multicolumn{1}{|c||}{} & \multicolumn{9}{c||}{ AM-MAE(dB) } & \multicolumn{6}{|c|}{ FM-MAE(dB) } \\
\hline SNR & T1 & T2 & T3 & T4 & T5 & T6 & T1 & T2 & T3 & T4 & T5 & T6 \\
\hline 4 & -16 & +07 & -16 & -16 & -16 & -16 & -30 & -33 & -33 & -32 & -39 & -33 \\
\hline 8 & -20 & +07 & -20 & -20 & -20 & -20 & -33 & -36 & -36 & -35 & -42 & -36 \\
\hline 12 & -24 & -10 & -24 & -24 & -24 & -24 & -38 & -40 & -40 & -39 & -45 & -40 \\
\hline 16 & -28 & -26 & -28 & -28 & -28 & -28 & -43 & -44 & -44 & -43 & -50 & -44 \\
\hline 20 & -32 & -31 & -32 & -32 & -32 & -32 & -48 & -49 & -49 & -47 & -54 & -49 \\
\hline 24 & -36 & -35 & -36 & -36 & -36 & -36 & -52 & -53 & -53 & -51 & -56 & -53 \\
\hline 28 & -40 & -39 & -40 & -40 & -40 & -40 & -56 & -57 & -57 & -56 & -58 & -57 \\
\hline 32 & -43 & -42 & -43 & -43 & -43 & -43 & -57 & -59 & -59 & -58 & -58 & -59 \\
\hline
\end{tabular}

absence of noise, the effect of parameters such as carrier frequency and frequency deviation was studied. The effect of bancipass white Gaussian noise on the performance of the AM-FM estimation techniques was also studied. The observations and conclusions were given at the end of each experiment

\section{REFERENCES}

[1] T.F. Quatieri, T.E. Hanna and G.C. O'Leary, "AM-FM separation using Auditory-Motivated Filters", IEEE Trans. Speech and Audio Pmc.. vol. 5, pp.465-480. Sept 1997.

[2] P. Maragos, J.F. Kaiser, and T.F. Quatien, "Energy Separation in Signal Modulations with Application to Speech Analysis" IEEE Trans. Signal Pmc.. vol. 41, No. 10,pp.3024-3051, Oct 1993.

[3] P.J. Loughlin and B. Tacer, "On the amplitude- and frequency- modulation decomposition of signals" $J$. Acoust. Soc. Am. 100(3), pp.1594-1601, Sep 1996.

[4] D. Vakman, "On the Analytical Signal, the TeagerKaiser Energy Algorithm, and other Methods for Defining Amplitude and Frequency" IEEE Trans. Signal Pmc. vol. 44, No. 4, pp.791-797. Apr 1996.

[5] L. Cohen, Erne-Frequency Analysis, Prentice Hall, New Jersey, 1995.

[6] P.J. Loughlin, J.W. Pitton, and L.E. Atlas, "Construction of Positive Time-frequency Distributions". IEEE Trans. Signal Proc.,vol. 42, No. 10, pp.2697-2705, Oct 1994. 